\title{
Involvement of xanthine oxidase and paraoxonase 1 in the process of oxidative stress in nonalcoholic fatty liver disease
}

\author{
BING WANG $^{1}$, RUI-NING YANG ${ }^{1}$, YUE-RONG ZHU ${ }^{1}$, JI-CHENG XING $^{1}$, \\ XIAO-WEI LOU ${ }^{1}$, YU-JIE HE ${ }^{1}$, QI-LONG DING ${ }^{2}$, MING-YUE ZHANG ${ }^{3}$ and HONG QIU ${ }^{1}$ \\ ${ }^{1}$ Department of Clinical Laboratory, PLA 81 Hospital, Nanjing, Jiangsu 210002; \\ ${ }^{2}$ Experimental and Teaching Center of Medical Basis for Pharmacy, China Pharmaceutical University, Nanjing, \\ Jiangsu 210009; ${ }^{3}$ Department of Blood Transfusion, Central Hospital of Nanyang, Nanyang, Henan 473000, P.R. China
}

Received November 19, 2015; Accepted November 14, 2016

DOI: $10.3892 / \mathrm{mmr} .2016 .6025$

\begin{abstract}
Xanthine oxidase (XOD) and paraoxonase 1 (PON1) are important enzymes in redox reactions in vivo, and are predominantly synthesized by the liver. The aim of the present study was to investigate the redox state in nonalcoholic fatty liver disease, and determine the association between the activities of XOD and PON1 and the severity of NAFLD. Sprague-Dawley rats were randomly divided into control, model and $\alpha$-lipoic acid (high and low dose) groups. The rats in the NAFLD model were induced by feeding a high fat diet for 12 weeks and the in vitro cell model of hepatocyte steatosis was induced by treating L- 02 cells with oleic acid for $24 \mathrm{~h}$. The body weight, liver function, lipid and oxidative stress indices, and histological features of the liver were examined in the rats. Compared with the control group, the rats in the NAFLD model group showed impaired liver function, lipid disorders and damage from oxidative stress. The serum activity of XOD increased significantly from the 4th week and was markedly higher, compared with that in the control group, reaching a peak in the 12th week. The activity of PON1 was negatively correlated with that of XOD. Compared with the control cells, the activity of XOD and levels of free-fatty acids were significantly higher, and the activity of PON1 was significantly lower in the NAFLD L-02 cell model. All the above indicators were significantly improved by treatment with the antioxidant, $\alpha$-lipoic acid. The activities of XOD and PON1 may be promising as markers in a noninvasive approach for detecting the severity of NAFLD clinically. $\alpha$-lipoic acid had protective effects on the NAFLD rats, and the potential
\end{abstract}

Correspondence to: Professor Hong Qiu, Department of Clinical Laboratory, PLA 81 Hospital, 34 Biao, Yanggongjing Street, Nanjing, Jiangsu 210002, P.R. China

E-mail: mapleqh@126.com

Key words: nonalcoholic fatty liver disease, xanthine oxidase, paraoxonase 1, oxidative stress mechanism may be associated with the inhibition of oxidative stress and lipid peroxidation.

\section{Introduction}

Nonalcoholic fatty liver disease (NAFLD) is an oxidative stress-associated liver lesion, which is caused by abnormal lipid metabolism, eliminating excessive drinking and other specific factors causing liver damage. At present, the incidence of NAFLD is increasing and at increasingly younger ages (1). This increase appears to be pandemic, as it is beginning to affect populations in developing countries due to the increased western lifestyle (2). NAFLD has become an important factor in chronic liver injury following viral hepatitis (3). The pathogenesis of NAFLD remains to be fully elucidate, however, it is closely associated with hepatic lipid deposition, high levels of reactive oxygen species (ROS) and oxidative stress damage caused by ROS $(4,5)$.

In the formation of NAFLD, the redox state is out of balance causing the associated oxidoreductases in tissues and body fluids to alter correspondingly (6), which includes xanthine oxidase (XOD) and paraoxonase 1 (PON1). XOD is an important enzyme in the metabolism of nucleic acids in the body, which are released into the circulation ahead of the enzyme, alanine aminotransferase (ALT), when liver injury occurs. XOD can catalyze xanthine, which cannot be further decomposed or metabolized, and accumulates due to liver cell damage, leading to an oxidation reaction and the production of peroxides. XOD is important in the generation of free radicals (7). Serum concentrations of XOD directly reflect the quantity of free radicals generated in liver tissue, and the increase in free radicals is important in the pathogenesis and development of NAFLD (8).

PON1 is an antioxidant enzyme synthesized and secreted by the liver, which can hydrolyze lipid peroxides. PON1 has a potent antioxidant function through specific binding with high-density lipoprotein (HDL). It is significant in protection against atherosclerosis, coronary heart disease and diabetes (9-11). Studies of liver disease have shown that PON1 can inhibit lipid peroxidation, target inflammation, relieve the progress of liver fibrosis and reduce the degree of liver damage by reducing hepatic oxidative stress $(12,13)$. The activity 
of PON1 in the serum is decreased significantly when liver damage occurs $(14,15)$.

At present, there are few reports on alterations of XOD and PON in NAFLD. The present study hypothesized that the activities of XOD and PON1 reflect the oxidation and antioxidant capacities in the body, namely the severity of oxidative stress injury, which indirectly reflects the severity of NAFLD. In the present study, an NAFLD rat model was established by feeding rats with a high fat diet (HFD), and an in vitro NAFLD model was established by treating L-02 human hepatocyte cells with oleic acid (OA), with $\alpha$-lipoic acid ( $\alpha$-LA) used for preventative intervention in the NAFLD models. The present study aimed to observe alterations in the activities of XOD and PON1 during modeling, examine the preventive and therapeutic effects of $\alpha$-LA on the NAFLD model in vivo and in vitro, and determine the relevance between the activities of XOD and PON1 and the severity of NAFLD.

\section{Materials and methods}

Chemicals and reagents. The assay kits used for measuring the serum levels of ALT, aspartate transaminase (AST), total cholesterol (TC), triglycerides (TG), HDL cholesterol (HDL-C), low-density lipoprotein cholesterol (LDL-C), free fatty acids (FFAs), and liver concentrations of TC, TG and FFAs, were purchased from Wako Pure Chemical Industries, Ltd. (Osaka, Japan). The assay kits for measuring XOD, malondialdehyde (MDA), superoxide dismutase (SOD), catalase (CAT), glutathione (GSH), glutathione peroxidase (GPX) and total antioxidant capacity (TAOC) were purchased from Nanjing Jiancheng Bioengineering Institute (Nanjing, China). Phenylacetate was purchased from Aladdin Reagent Co., Ltd (Shanghai, China). OA and Oil Red O were purchased from Sigma-Aldrich; Merck Millipore (Darmstadt, Germany). $\alpha$-LA was purchased from STADA (Dresden, Germany). 3-(4,5-Dimethylthiazole-2yl)-2,5-diphenyltetrazolium bromide (MTT) reagent was purchased from Roche Diagnostics GmbH (Mannheim, Germany). DMEM culture medium was purchased from Gibco (Thermo Fisher Scientific, Inc., Waltham, MA, USA).

Animals and treatment. A total of 32 male Sprague-Dawley rats, weighing 180-200 g, were supplied by Jiangsu University Laboratory Animal Center (Jiangsu, China). The animals were maintained at an ambient temperature of $23.0 \pm 2^{\circ} \mathrm{C}$ and the natural circadian rhythm of light. The rat model of NAFLD was established by feeding the rats with a HFD) according to our previous study (16). Briefly, following acclimation for 1 week, the animals were randomly divided into four groups ( 8 rats per group): Control, model, $\alpha$-LA high dose $\left(\alpha-\mathrm{LA}_{\mathrm{H}}\right)$ and $\alpha$-LA low dose $\left(\alpha-\mathrm{LA}_{\mathrm{L}}\right)$. The rats in the control group were fed a standard diet and rats in the other three groups were fed a HFD for 12 weeks. The rats in the $\alpha-\mathrm{LA}_{\mathrm{H}}$ and $\alpha-\mathrm{LA} \mathrm{A}_{\mathrm{L}}$ groups were administered with $\alpha$-LA solution intragastrically at doses of 20 and $40 \mathrm{mg} / \mathrm{kg} / \mathrm{day}$, respectively. The rats in the control and model groups were administered with the same volume of normal saline by intragastric administration. The rats were weighed twice per week. At the end of weeks 4 , $6,8,10$ and 12 , the rats were anesthetized with etherization, and blood was collected from the fundus oculi venous plexus.
Serum was isolated $\left(1,000 \mathrm{x} \mathrm{g}\right.$, centrifuged for $10 \mathrm{~min}$, at $\left.25^{\circ} \mathrm{C}\right)$ and frozen at $-80^{\circ} \mathrm{C}$. At the end of the experiment, the rats were sacrificed by cervical dislocation and entire livers were collected and weighed. The liver index (LI) was calculated as follows: LI=liver weight $(\mathrm{g}) /$ body weight(BW, g)x $100 \%$. Certain sections of the liver tissue in the same positions were immersed in $10 \%$ buffered formalin solution for histological examination. The remaining tissues were frozen at $-80^{\circ} \mathrm{C}$ for the production of hepatic tissue homogenate.

NAFLD cell model construction. The L-02 cells were provided by the Experimental Teaching Center of Medical Basis for Pharmacy, China Pharmaceutical University (Nanjing, China). The in vitro NAFLD model was established by treating the L-02 cells with OA. The L-02 cells were seeded at a density of $5 \times 10^{5}$ cells/well in six-well plates and incubated for $24 \mathrm{~h}$ at $37^{\circ} \mathrm{C}, 5 \% \mathrm{CO}_{2}$ in DMEM culture medium. The cells were switched to serum-free media containing $60 \mu \mathrm{g} / \mathrm{ml} \mathrm{OA}$ and incubated for a further $24 \mathrm{~h}$. The cells were stained using Oil Red $\mathrm{O}$ and examined for lipid accumulation by inverted microscope (Nikon Corporation, Tokyo, Japan).

The L-02 cells were seeded into six-well plates at a concentration of $5 \times 10^{5}$ cells/well and were incubated for an initial $24 \mathrm{~h}$. The cells then were divided into the following three groups: Control, model and $\alpha$-LA, and incubated for another $24 \mathrm{~h}$. The control cells were switched to DMEM containing $2 \%$ fetal bovine serum (FBS) at $37^{\circ} \mathrm{C}, 5 \% \mathrm{CO}_{2}$. The model cells were switched to media containing $2 \% \mathrm{FBS}$ and $60 \mu \mathrm{g} / \mathrm{ml}$ OA. The $\alpha$-LA-treated cells were switched to media containing $2 \% \mathrm{FBS}, 60 \mu \mathrm{g} / \mathrm{ml} \mathrm{OA}$ and 50, 100, 200 or $400 \mu \mathrm{mol} / 1 \alpha$-LA, respectively. Cell viability was determined using an MTT assay and examined by measuring the absorbance at $490 \mathrm{~nm}$. The cells and culture supernatants of all groups were collected for FFA, XOD and PON1 determination.

Determination of biochemical indicators and redox indices. The serum levels of ALT, AST, TC, TG, HDL-C, LDL-C and FFAs were determined using a 7600 Automatic Biochemistry Analyzer (Hitachi, Tokyo, Japan) in accordance with the manufacturer's protocols. The serum enzymatic activities of XOD, PON1, SOD, CAT and GPX, and the levels of TAOC, MDA and GSH were determined using a colorimetric method with commercial kits. The activity of the PON1 enzyme was evaluated using phenylacetate as a substrate to determine arylesterase activity. Briefly, the arylesterase activity was measured using $1 \mathrm{mmol}$ phenylacetate (Aladdin, Beijing, China) as the substrate in $20 \mathrm{mmol} / \mathrm{l}$ Tris-HCl buffer ( $\mathrm{pH} 8.0$ ) with $1 \mathrm{mmol} / 1 \mathrm{CaCl}_{2}$; the activity was expressed as $\mathrm{kU} / 1$.

Preparation of the liver homogenates was performed on ice. The liver tissues were homogenized in ice-chilled $0.9 \% \mathrm{NaCl}$ to yield $10 \%(\mathrm{w} / \mathrm{v})$ homogenate and centrifuged at $1,000 \mathrm{x} \mathrm{g}$ for $10 \mathrm{~min}$ at $4^{\circ} \mathrm{C}$, with the resulting supernatant used for the detection of TC, TG and FFAs, and the XOD, PON1, SOD, CAT, GPX, TAOC, MDA and GSH redox indices.

Histological examination. The liver tissues were fixed in $10 \%$ formaldehyde solution, embedded in paraffin, sliced into $5 \mu \mathrm{m}$ thick sections, and stained with hematoxylin and eosin (H\&E). Two pathologists performed the assessment of fatty degeneration and focal necrosis independently. Pathological evaluations 
Table I. Alterations in the BW and LI of rats.

\begin{tabular}{lllll}
\hline & \multicolumn{3}{c}{ BW $(\mathrm{g})$} & \\
\cline { 2 - 5 } Group & \multicolumn{1}{c}{ Week 4 } & Week 8 & Week 12 LI (\%) & \\
\hline Control & $327.00 \pm 8.49$ & $425.63 \pm 18.21$ & $471.88 \pm 30.85$ & $2.67 \pm 0.20$ \\
Model & $385.63 \pm 26.34^{\mathrm{b}}$ & $461.75 \pm 39.73^{\mathrm{a}}$ & $514.13 \pm 46.10^{\mathrm{a}}$ & $4.04 \pm 0.16^{\mathrm{b}}$ \\
$\alpha-\mathrm{LA}_{\mathrm{L}}$ & $306.88 \pm 11.46^{\mathrm{c}}$ & $399.13 \pm 30.613^{\mathrm{c}}$ & $421.63 \pm 27.26^{\mathrm{c}}$ & $3.55 \pm 0.25^{\mathrm{c}}$ \\
$\alpha-\mathrm{LA}_{\mathrm{H}}$ & $297.38 \pm 18.62^{\mathrm{c}}$ & $364.13 \pm 14.10^{\mathrm{c}}$ & $375.63 \pm 15.61^{\mathrm{c}}$ & $3.17 \pm 0.21^{\mathrm{c}}$ \\
\hline
\end{tabular}

$\mathrm{LI} / \mathrm{BW} \times 100 \%$. Data are presented as the mean \pm standard deviation $(\mathrm{n}=8) .{ }^{\mathrm{a}} \mathrm{P}<0.05$ and ${ }^{\mathrm{b}} \mathrm{P}<0.01$, vs. control group; ${ }^{\mathrm{c}} \mathrm{P}<0.01$, vs. model group BW, body weight; LI, liver index; $\alpha$-LA , low-dose $\alpha$-lipoic acid; $\alpha$-LA $A_{H}$, high-dose $\alpha$-LA.

by optical microscope (Olympus Corporation, Tokyo, Japan) were based on lobular inflammation, necrosis, which was scored as follows: 0,0 foci; $1,<2$ foci; $2,2-4$ foci; and 3, $>4$ foci per field (magnification, $\mathrm{x} 200$ ), and steatosis, which was scored as follows: $0,<5 \% ; 1,6-33 \% ; 2,34-66 \%$; and $3,>66 \%$.

Oil Red $O$ staining. The L-02 cell monolayers were gently rinsed twice with phosphate-buffered saline, fixed with $60 \%$ isopropanol for $1 \mathrm{~min}$ at room temperature, stained with $0.5 \%$ Oil Red O-isopropanol for $10 \mathrm{~min}$ and then washed with distilled water. The cells were visualized using a bright-field optical microscope (CX-41; Olympus Corporation, Tokyo, Japan). Three typical high-power fields were selected at random and the integral optical density (IOD) of the red lipid droplets was analyzed using ImagePro Plus 6.0 software (Media Cybernetics, Inc., Rockville, MD, USA).

Statistical analysis. SPSS 16.0 statistical software (SPSS, Inc., Chicago, IL, USA) was used for statistical analysis. All values are expressed as the mean \pm standard deviation. Comparison of different groups was performed using Student's t-test and one-way analysis of variance. $\mathrm{P}<0.05$ was considered to indicate a statistically significant difference.

\section{Results}

Alterations in the BWs and LIs of rats. With the extension of the duration of HFD administration, the BWs and LIs of rats in the model group were significantly higher, compared with those in the controls $(\mathrm{P}<0.01 / \mathrm{P}<0.05)$. $\alpha$-LA significantly decreased the BWs of the rats, compared with those in model group $(\mathrm{P}<0.01$; Table I).

Alterations in serum liver function and lipid indices. The ingestion of HFD by the rats in the model group resulted in significant increases in ALT, AST, TC, TG, LDL-C and FFAs, and a decrease in the level of HDL-C, compared with the rats in the control group $(\mathrm{P}<0.01 / \mathrm{P}<0.05)$. The serum levels of ALT, AST, TC, TG and FFAs in the rats in the $\alpha$-LA group were significantly lower, compared with those in the model rats, and the levels of HDL-C and LDL-C in the $\alpha$-LA group were higher and lower, respectively, compared with those in the model rats, although without statistical significance (Fig. 1A and B).
Alterations in hepatic lipids. The intrahepatic concentrations of TC, TG and FFAs in the model rats were significantly higher, compared with those in the control rats $(\mathrm{P}<0.01)$, and were significantly lower in the $\alpha$-LA rats, compared with the model rats $(\mathrm{P}<0.01)$. These results showed that $\alpha$-LA improved hepatic lipid metabolism (Fig. 1C).

Alterations in the activities of XOD and PON1. Compared with the rats in the control group, the serum activity of XOD was significantly higher and that of PON1 was significantly lower in the rats in the model group $(\mathrm{P}<0.01)$. With the increased duration of HFD feeding, these alterations became gradually more marked. In the model group, there were significant differences between the week 4, 6, 8, 10 and $12 \mathrm{XOD} / \mathrm{PON} 1$ activities $(\mathrm{P}<0.01)$. The serum activities of XOD and PON1 in the $\alpha$-LA group rats were significantly lower and higher, respectively, compared with those in the model group rats $(\mathrm{P}<0.01$; Fig. 2A and B). The hepatic tissue activities of XOD and PON1 were also measured. The HFD significantly increased the hepatic activity of XOD and decreased the hepatic activity of PON1, compared with the control group $(\mathrm{P}<0.01)$, and $\alpha$-LA returned the activities of XOD and PON1 to normal (Fig. 2C).

Alterations in redox indices. The oxidative stress statuses of the rats were evaluated by analyzing the serum and tissue levels of SOD, CAT, GPX, TAOC, MDA and GSH. Compared with the control group rats, the serum and tissue levels of SOD, CAT, GPX, TAOC and GSH were significantly lower, and the level of MDA was significantly higher in the model group rats $(\mathrm{P}<0.01)$. Compared with the model group rats, the serum and tissue levels of SOD, CAT, GPX, TAOC and GSH were significantly higher, and the level of MDA was significantly lower in the $\alpha$-LA group rats $(\mathrm{P}<0.01$; Fig. $3 \mathrm{~A}-\mathrm{F})$.

Histological observations. Macroscopic examination of the livers of the rats in the control group showed a normal red, smooth and shiny appearance. By contrast, the livers from the model group were yellowish brown, enlarged and greasy. The livers from the $\alpha$-LA group showed a more normal appearance, and the size and texture of the livers in the $\alpha$-LA group were intermediary between those of the control group and model group. Examination of the liver tissues using light microscopy in the tissues of the control group stained with $\mathrm{H} \& \mathrm{E}$ showed normal polyhedral hepatocytes with central 
A

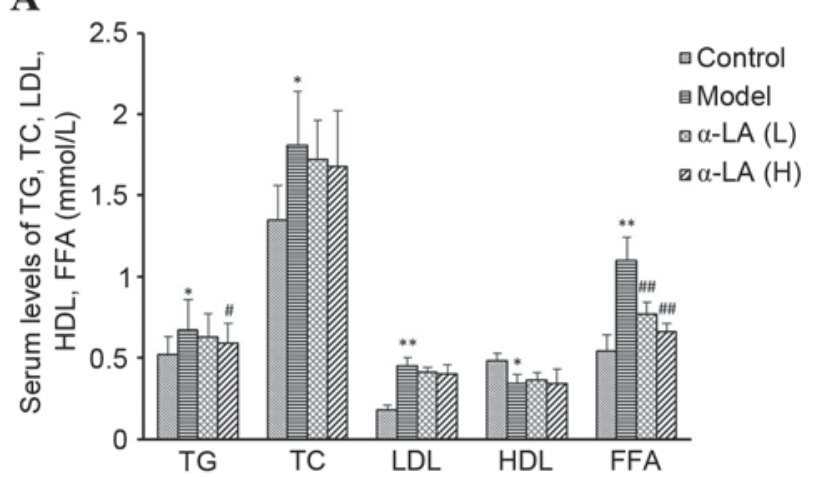

B

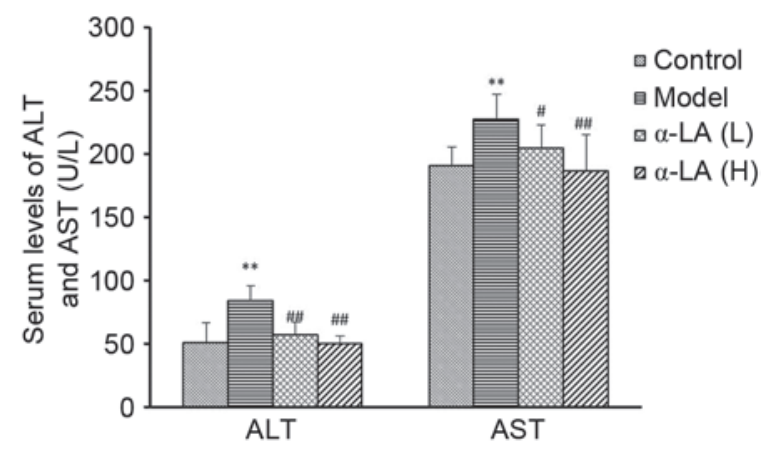

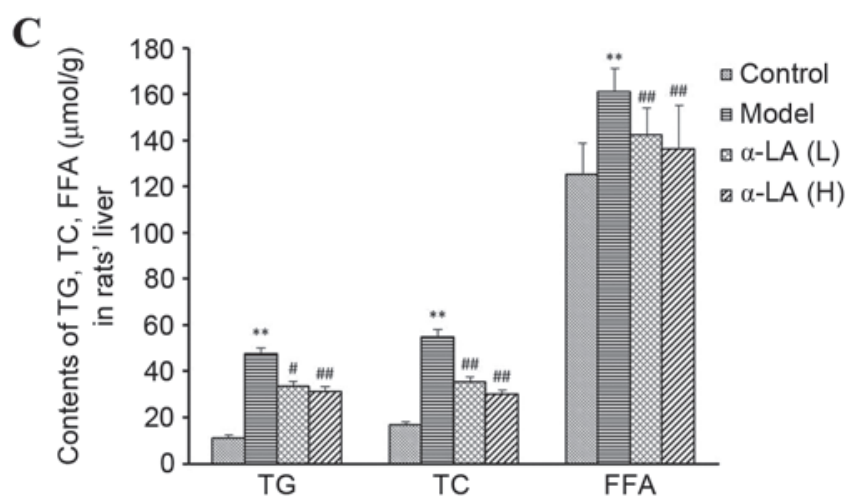

Figure 1. Alterations in serum levels of ALT, AST, TC, TG, HDL, LDL and FFAs, and intrahepatic TG, TC, and FFA content. After 12 weeks of feeding a high fat diet, liver function and serum lipids of Sprague-Dawley rats were detected. (A) Levels of TC, TG, HDL, LDL and FFAs in serum. (B) Levels of ALT, AST in serum. (C) Levels of TC, TG and FFAs in liver tissues. ${ }^{*} \mathrm{P}<0.05$ and ${ }^{* *} \mathrm{P}<0.01$, vs. control group; ${ }^{*} \mathrm{P}<0.05$ and ${ }^{\# \#} \mathrm{P}<0.01$, vs. model group. ALT, alanine aminotransferase; AST, aspartate transaminase; TC, total cholesterol; TG, triglycerides; HDL, high-density lipoprotein; LDL, low-density lipoprotein; FFAs, free fatty acids.

A

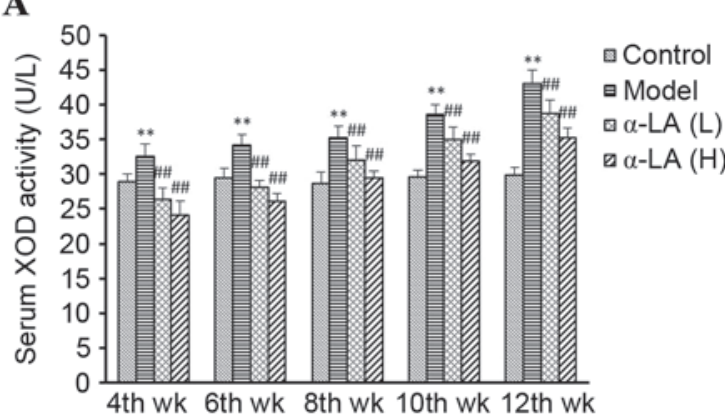

B

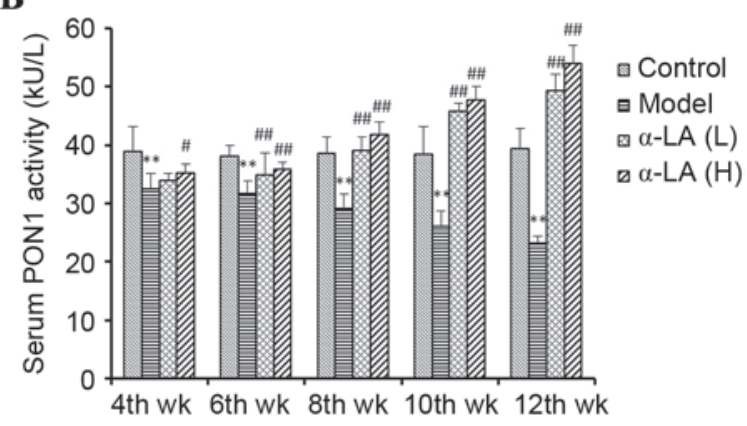

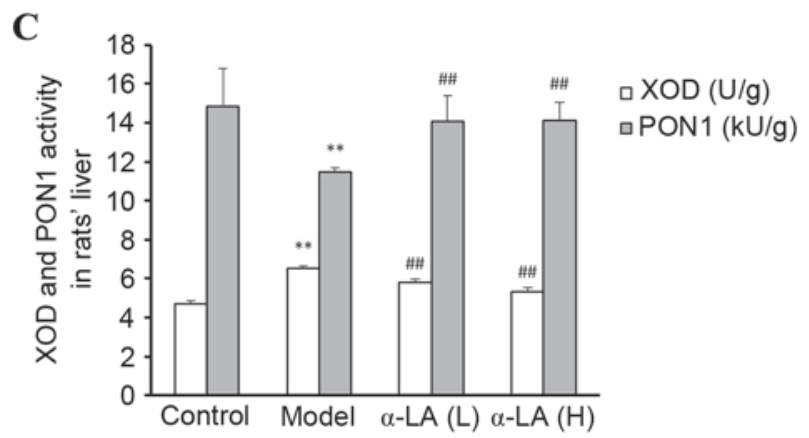

Figure 2. Alterations in activities of XOD and PON1 in serum and hepatic tissue. At the end of weeks 4, 6, 8, 10 and 12, serum samples from each group were collected and activities of (A) XOD and (B) PON1 were detected. (C) After 12 weeks of feeding high fat diet, rats were sacrificed and the activities of XOD and PON1 in liver tissues were detected. ${ }^{* *} \mathrm{P}<0.01$ vs. control group; ${ }^{\#} \mathrm{P}<0.05$ and ${ }^{\# \#} \mathrm{P}<0.01$, vs. model group. $\mathrm{XOD}$, xanthine oxidase; PON1, paraoxonase 1.

nuclei and an eosinophilic cytoplasm (Fig. 4A). Compared with the rats in the control group with a normal liver histology, the rats in the model group developed various degrees of diffuse hepatic steatosis, characterized by the accumulation of 
$\mathbf{A}$

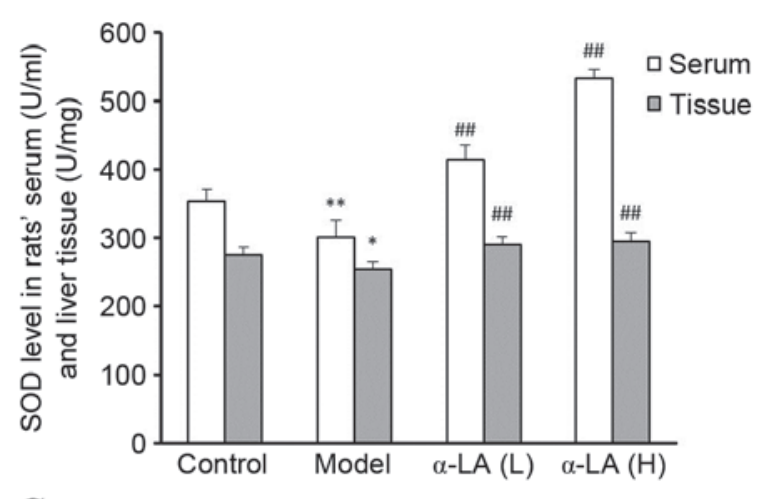

C

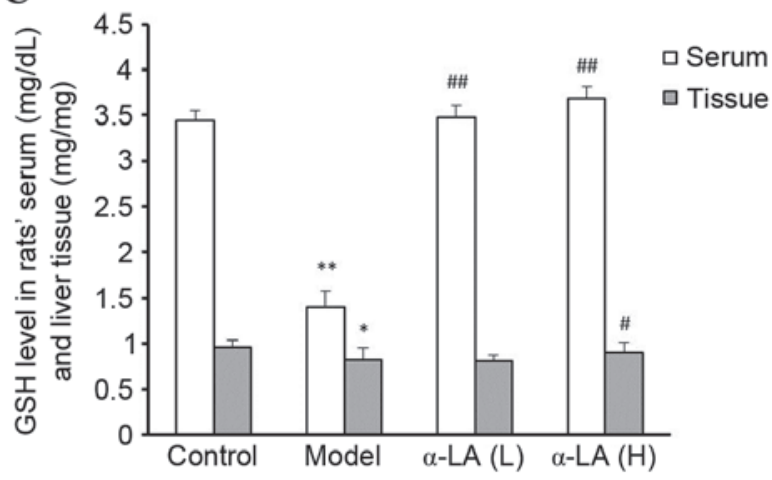

$\mathbf{E}$

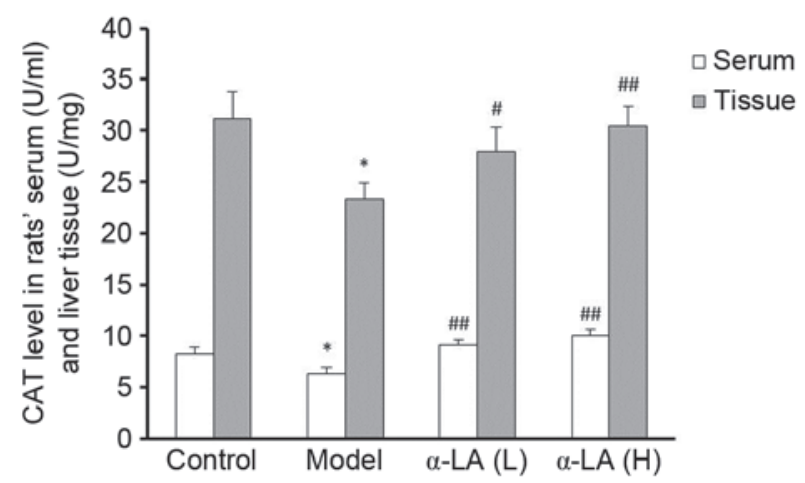

B

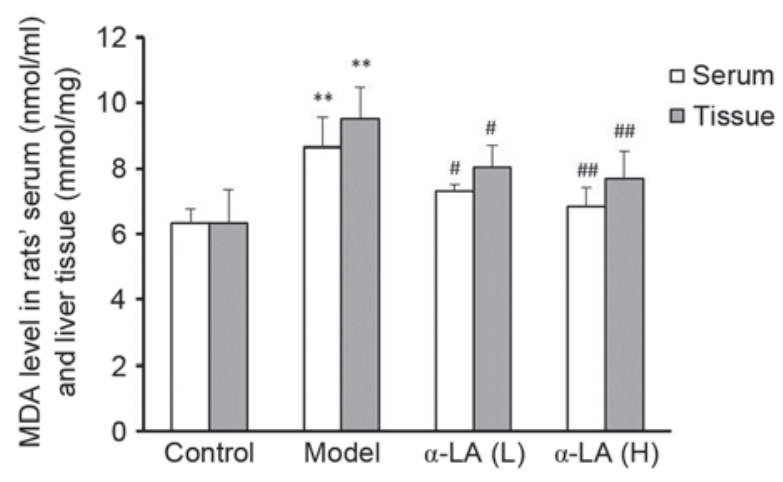

D

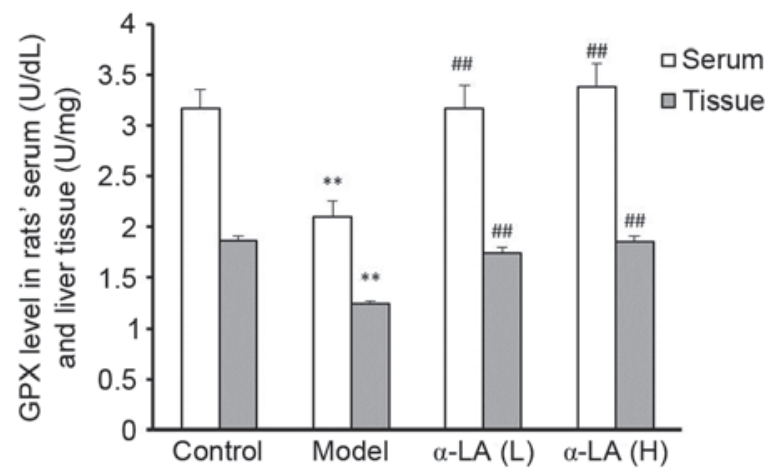

$\mathbf{F}$

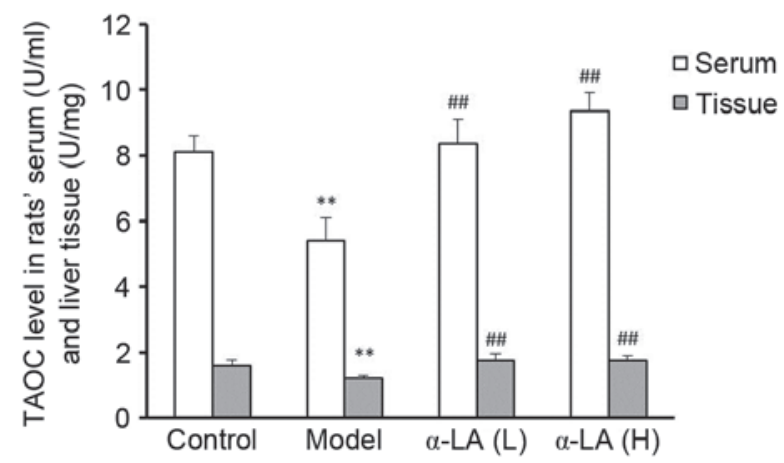

Figure 3. Alterations in the levels of (A) SOD, (B) MDA, (C) GSH, (D) GPX, (E) CAT and (F) TAOC in serum and tissue. After 12 weeks of feeding a high fat diet, serum and liver tissues of each group were collected and the levels of SOD, CAT, GPX, TAOC, MDA and GSH were detected. "P<0.05 and ${ }^{* *} \mathrm{P}<0.01$, vs. control group; ${ }^{*} \mathrm{P}<0.05$ and ${ }^{\# \#} \mathrm{P}<0.01$, vs. model group. SOD, superoxide dismutase; CAT, catalase; GSH, glutathione; GPX, glutathione peroxidase; TAOC, total antioxidant capacity; MDA, malondialdehyde.

fat, hepatocyte ballooning, loss of cytoplasmic eosin, eccentric nuclei, intralobular inflammation and necrosis (Fig. 4B). $\alpha$-LA treatment markedly attenuated the histopathological characteristics of NAFLD observed in the model group. There was mild microvesicular steatosis in the tissues, and the steatosis was almost absent in the remainder of the samples, with intact architecture and no inflammatory foci. Compared with the $\alpha-\mathrm{LA}_{\mathrm{L}}$ group (Fig. $4 \mathrm{C}$ ), the improvement was more marked in the $\alpha-\mathrm{LA}_{\mathrm{H}}$ group (Fig. 4D). The histological data showed that the model group exhibited grade 2-3 focal necrosis and grade 1-2 hepatic steatosis, whereas the normal control group exhibited grade $0-1$ focal necrosis and grade 0 hepatic steatosis (Table II).

OA induces lipid accumulation in L-02 cells. The L-02 cells were incubated in DMEM containing $60 \mu \mathrm{g} / \mathrm{ml} \mathrm{OA} \mathrm{mixture}$ for $24 \mathrm{~h}$ and were then stained with Oil Red O. A substantial quantity of red lipid droplets was observed in the L-02 cells of the model group (Fig. 5A). However, no lipid droplets were detected in the L-02 cells of the control group (Fig. 5A). As shown in Fig. 5B, the IOD value in the model cells was significantly higher, compared with that in the control cells $(\mathrm{P}<0.01)$. The cytotoxicity of OA towards the L-02 cells was analyzed using an MTT assay. The results revealed no significant difference between the control group and the model group in terms of cell viability ( $\mathrm{P}>0.01$; Fig. $5 \mathrm{C})$.

a-LA reduces the accumulation of FFAs in NAFLD model cells. To determine the effect of $\alpha$-LA on FFA accumulation in the OA-treated L-02 cells, the L-02 cells were treated in different groups, and the extracellular and intracellular FFAs were quantified. Compared with the control cells, the levels of 
A

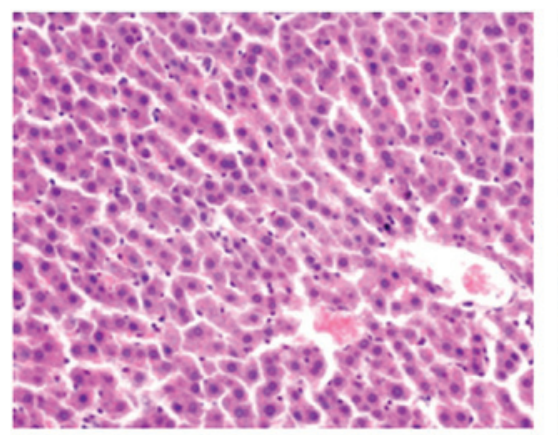

C

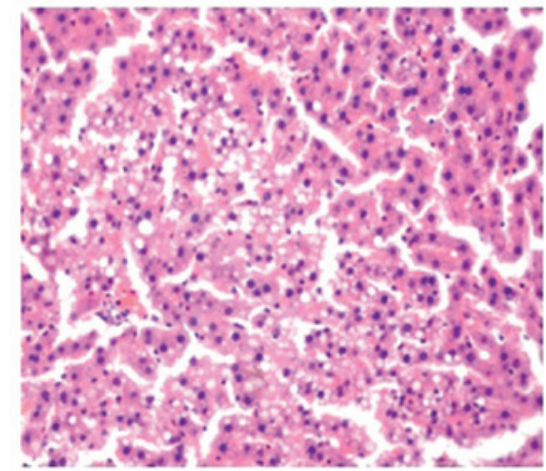

B

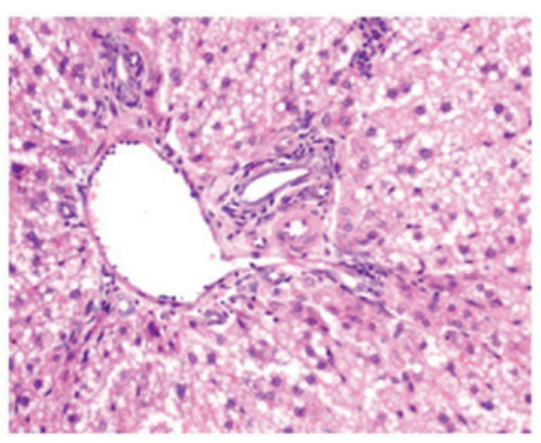

D

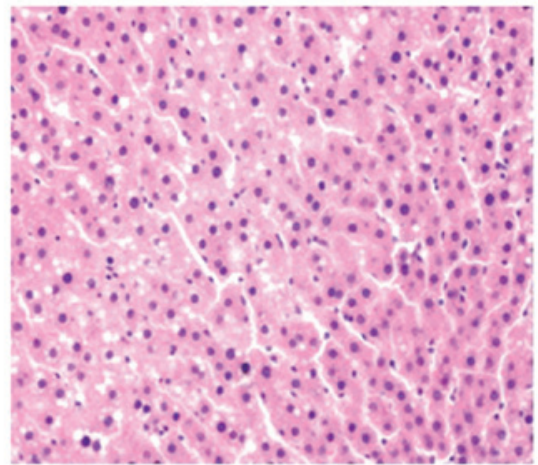

Figure 4. Histological alterations in the (A) control, (B) model, (C) $\alpha-\mathrm{LA}_{\mathrm{L}}$ and (D) $\alpha$-LA $\mathrm{H}_{\mathrm{H}}$ groups. Hematoxylin and eosin staining was used (original magnification, $\mathrm{x} 400$ ). $\alpha$-LA, $\alpha$-lipoic acid.

A

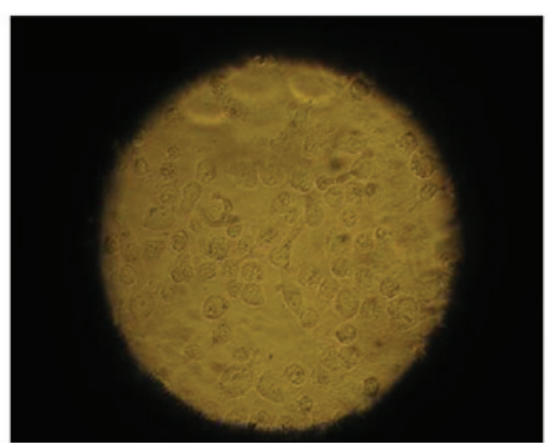

Control

B

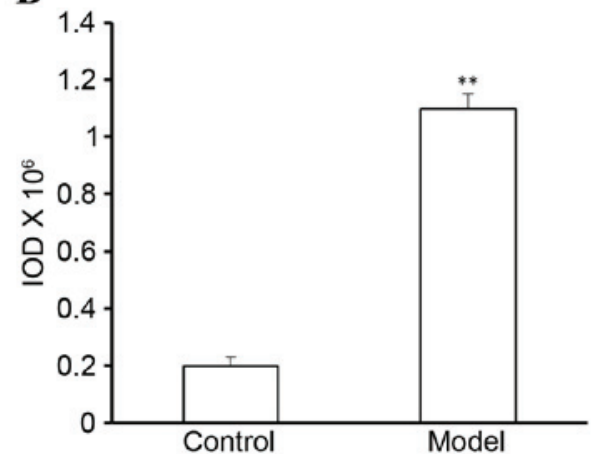

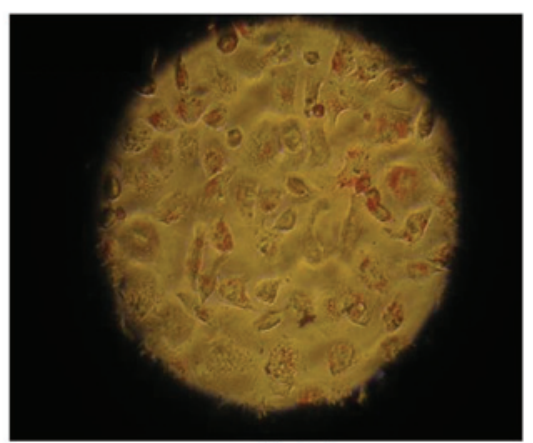

Model

C

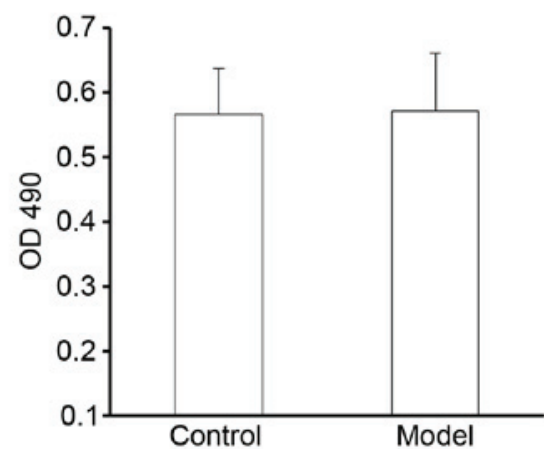

Figure 5. Lipid accumulation in L-02 cells. L-02 cells were induced by DMEM containing $60 \mu \mathrm{g} / \mathrm{ml}$ oleic acid mixture for 24 h. (A) Oil Red O staining in the control and model group. Magnification, x400. (B) IOD values were counted and (C) cell viability was detected by measuring the absorbance at 490 nm. ${ }^{* *} \mathrm{P}<0.01$, vs. control group. IOD, integral optical density; OD, optical density.

extracellular and intracellular FFAs were significantly higher following treatment with $60 \mu \mathrm{g} / \mathrm{ml} \mathrm{OA}(\mathrm{P}<0.01)$. Compared with the cells in the model group, $\alpha$-LA significantly reduced the FFA concentrations ( $\mathrm{P}<0.01$; Fig. 6A). 
Table II. Summary of histological scoring for liver tissue sections of different treatment groups.

\begin{tabular}{lcc}
\hline Group & Focal necrosis & Hepatic steatosis \\
\hline Control & $0.125 \pm 0.35$ & 0 \\
Model & $2.75 \pm 0.46^{\mathrm{a}}$ & $1.63 \pm 0.52^{\mathrm{a}}$ \\
$\alpha-\mathrm{LA}_{\mathrm{L}}$ & $2.00 \pm 0.53^{\mathrm{b}}$ & $1.00 \pm 0.53^{\mathrm{b}}$ \\
$\alpha-\mathrm{LA}_{\mathrm{H}}$ & $1.63 \pm 0.52^{\mathrm{c}}$ & $0.63 \pm 0.52^{\mathrm{c}}$ \\
\hline
\end{tabular}

Hematoxylin and eosin staining was used to stain liver tissues in the control, model, $\alpha-\mathrm{LA}_{\mathrm{L}}$, and $\alpha-\mathrm{LA}_{\mathrm{H}}$ groups. Data are presented as the mean \pm standard deviation $(\mathrm{n}=8) .{ }^{\mathrm{a}} \mathrm{P}<0.01$, vs. control group; ${ }^{\mathrm{b}} \mathrm{P}<0.05$ and ${ }^{\mathrm{C}} \mathrm{P}<0.01$, vs. model group. $\alpha$-LA $\mathrm{L}_{\mathrm{L}}$, low-dose $\alpha$-lipoic acid; $\alpha-\mathrm{LA}_{\mathrm{H}}$, high-dose $\alpha$-LA.

A

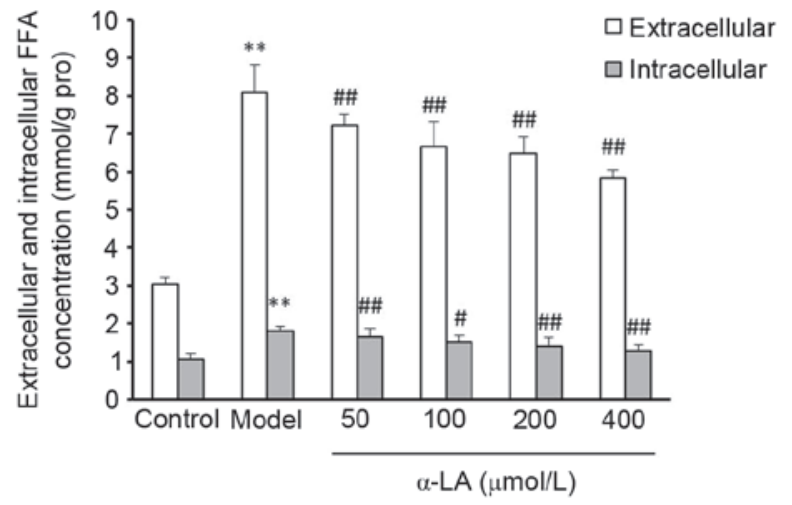

B

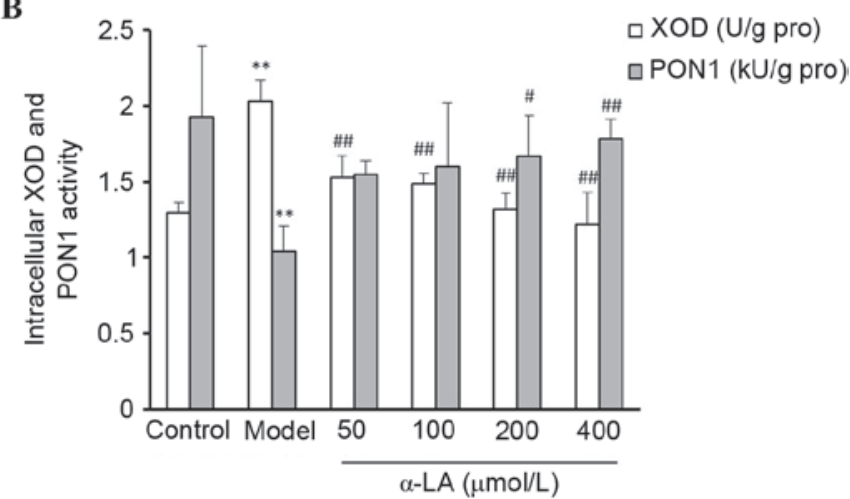

Figure 6. Alterations in the activities of FFAs, XOD and PON1 in L-02 cells. Control cells were incubated in media containing $2 \%$ FBS. Model cells were incubated in media containing $2 \%$ FBS and $60 \mu \mathrm{g} / \mathrm{ml}$ OA. $\alpha$-LA treated cells were switched to media containing $2 \% \mathrm{FBS}, 60 \mu \mathrm{g} / \mathrm{ml} \mathrm{OA}$ and 50,100 , 200 or $400 \mu \mathrm{mol} / 1 \alpha$-LA, respectively. (A) Extracellular and intracellular FFA activity. (B) Intracellular XOD and PON1 activity. ${ }^{* *} \mathrm{P}<0.01$. vs. control group; ${ }^{*} \mathrm{P}<0.05$ and ${ }^{\# \#} \mathrm{P}<0.01$, vs. model group. OA, oleic acid; $\alpha$-LA, $\alpha$-lipoic acid; FFAs, free fatty acids; XOD, xanthine oxidase; PON1, paraoxonase 1.

Alterations in the activities of XOD and PON1 in L-02 cells. As shown in Fig. 6B, compared with the control cells, the intracellular XOD activity was significantly higher and the activity of PON1 was significantly lower in the model cells $(\mathrm{P}<0.01)$. Compared with control cells, the intracellular XOD activity was significantly lower in the $\alpha$-LA treated cells $(\mathrm{P}<0.01)$ and the activity of PON1 was significantly higher, until the $\alpha$-LA concentration reached $200 \mu \mathrm{mol} / 1(\mathrm{P}<0.01)$.

\section{Discussion}

NAFLD is a clinical pathological syndrome without a history of excessive alcohol consumption, characterized by fatty denaturation and lipid accumulation in hepatocytes. Histopathologically, it exhibits the following characteristics: Simple fatty liver, steatohepatitis, fatty liver fibrosis and hepatocirrhosis, which may exist alone or in combination, and the clinical manifestations of NAFLD are diverse (17). The pathogenesis of NAFLD remains to be fully elucidated, and the current hypothesis of 'two hits' has been well accepted $(4,18)$. In the two hits hypothesis, insulin resistance is the basis of pathogenesis, and ROS are the core link of pathogenesis. The substantial quantity of ROS generated is beyond the removal capacity of the anti-oxidative system. Consequently, oxidative stress occurs together with substantial peroxide formation, and the peroxide leads to hepatocyte damage, which induce inflammatory reactions during which inflammatory cells infiltrate the liver parenchyma (19). Therefore, NAFLD occurs as a result of an imbalance between oxidative stress and resistance against oxidative stress, and results in imbalance of the redox system in the body. Oxidative stress persists in NAFLD and several studies have demonstrated that severe lipid peroxidation exist in patients with NAFLD $(20,21)$. It is important for clarification of the pathogenesis, diagnosis and sequelae of NAFLD to investigate alterations in oxidase and antioxidase, and to examine the redox state of the body. The findings of the experiments performed in the present study using an animal model of NAFLD confirmed this. It was found that the oxidative product, MDA, was generated in high levels, however, anti-oxidative capacity decreased substantially due to reductions in oxidants, including SOD, GSH and CAT. The alterations in these indices indicated that the NAFLD rat model had been successfully established. The state of oxidative stress in the body can be evaluated by detecting the metabolites in the associated tissues and body fluids during oxidative stress, which can assist in judging the severity and prognosis of NAFLD.

The tissue with the highest expression levels of XOD is the liver $(22,23)$. Usually, $90 \%$ of XOD is present in hepatocytes in the form of its precursor, xanthine dehydrogenase (XD), and is generally inactive. In cases of tissues in a pathological state, including oxidative stress, XD converts to XOD, the activity of which increases significantly. XOD then catalyzes the oxidation of xanthine; generating numerous free radicals, which mediate the peroxidation of lipids, and trigger the occurrence and progression of NAFLD (24). As the increase in the activity of XOD leads to the production of increased free radicals, the activity of XOD in the serum reflects the production of free radicals in tissues directly, and represents the degree of liver damage due to oxidative stress. The present study showed that an increase in peroxidative products and XOD activity in the NAFLD rats resulted from excess XOD, which was released into the blood circulation of NAFLD rats, causing the production of free radicals, which were involved in the onset and progression of NAFLD.

PON1 is an anti-oxidative enzyme, which the liver synthesizes and secretes, and has high levels of activity in the liver and the blood (25). PON1 can bind to HDL to inhibit oxidative modification (26), preventing against the accumulation of 
oxidative products on LDL (27) to reduce the lipid peroxidation-induced damage in endothelial cells (28), the production of peroxides and oxidative phosphatide, and the incidence of atherosclerosis (29). It had been found that activity of PON1 is negatively correlated with LDL-C and TG, and that the PON1 gene plasmid can increase the activity of PON1 in the serum, and inhibit lipid accumulation in the blood and the liver (30). If the PON1 gene plasmid is used to treat NAFLD, the activity of PON1 in the serum increases, whereas the activities of TC, LDL-C and TG are decreased to suppress lipid accumulation in the blood and the liver, mitigating the liver damage induced by a HFD. The results of the present study showed that the activity of PON1 decreased markedly in the serum of the NAFLD model rats, whereas the levels of TC, LDL-C and TG increased. It has also been found that LDL and HDL in the plasma are more readily oxidized in PON1-knockout mice (31). In NAFLD, reduced PON1 and increased MDA activity can be considered a biochemical marker for lipid peroxidation (14). PON1 may be closely associated with lipid peroxidation and oxidative stress.

NAFLD is a complex pathological procedure of chronic liver damage involving several factors, and oxidative stress is important in the occurrence and progression of NAFLD. The present study hypothesized that alterations in the activities of XOD and PON1 are associated with the severity of NAFLD. The results of the present study showed that the activities of XOD in the serum, tissues and cells of the model groups were higher, compared wit those in the control group, whereas the activities of PON1 were lower, compared with those in the control group. Notable fat denaturation and inflammatory necrosis were present in the liver tissues of the model group. Alterations in the activities of XOD and PON1 were time-dependent, and with increasing duration in the formation of the animal model, the activities of XOD and PON1 increased and decreased, respectively.

In the body, $\alpha$-LA is a sole water-soluble and fat-soluble antioxidant, and has the capacity to remove free radicals and resist oxidation (32-34), which has lead to it being applied widely in biomedicine and associated areas. Following the administration of $\alpha$-LA in the present study, the state of oxidative stress, indicated by the levels of SOD, CAT, GPX, TAOC, MDA and GSH, in the serum and liver tissues of the NAFLD rats were markedly relieved, the activities of XOD and MDA decreased significantly, and the activities of SOD and PON1 increased significantly, which indicated that $\alpha$-LA had a potent reversal effect on HFD-induced oxidative stress. The administration of $\alpha$-LA also improves lipid metabolism $(35,36)$. In the present study, $\alpha$-LA reduced the levels of TC, TG and FFAs in the serum and L-02 cells in the model groups. The results from the pathological sections of the liver also showed decreased lipid deposition in the hepatocytes, and relief of pathological damage of hepatocytes, suggesting that $\alpha$-LA had certain preventive effects. The available evidence suggested that $\alpha$-LA may delay the development of NAFLD, to a certain extent, by resisting oxidative stress and reducing the damage of lipid peroxidation.

In conclusion, in the occurrence and progression of NAFLD, oxidative stress usually persists. XOD and PON1 were found to represent a state of oxidative stress and anti-oxidative capacity in the body, and indirectly reflect the severity of NAFLD, to a certain extent. Despite debate regarding whether anti-oxidative therapy is effective in NAFLD $(37,38)$, the results of the present study showed that the antioxidant, $\alpha$-LA, led to an improvement in the symptoms of NAFLD. However, why the activities of XOD and PON1 alter NAFLD, and whether this alteration is associated with the expression levels of XOD and PON1 remain to be elucidated. Therefore, further investigations on the association between XOD and PON1 and the occurrence and progression of NAFLD are required, which may be useful for early diagnosis, treatment and prognosis of the disease.

\section{References}

1. Singer C, Stancu P, Coşoveanu S and Botu A: Non-alcoholic fatty liver disease in children. Curr Health Sci J 40: 170-176, 2014.

2. Masarone M, Federico A, Abenavoli L, Loguercio C and Persico M: Non alcoholic Fatty liver: Epidemiology and natural history. Rev Recent Clin Trials 9: 126-133, 2014.

3. Wang FS, Fan JG, Zhang Z, Gao B and Wang HY: The global burden of liver disease: The major impact of China. Hepatology 60: 2099-2108, 2014.

4. Mehta K, Van Thiel DH, Shah N and Mobarhan S: Nonalcoholic fatty liver disease: Pathogenesis and the role of antioxidants. Nutr Rev 60: 289-293, 2002.

5. Guo J, Ren W, Li A, Ding Y, Guo W, Su D, Hu C, Xu K, Chen H, $\mathrm{Xu}$ X, et al: Fat mass and obesity-associated gene enhances oxidative stress and lipogenesis in nonalcoholic fatty liver disease. Dig Dis Sci 58: 1004-1009, 2013.

6. Surapaneni KM and Jainu M: Comparative effect of pioglitazone, quercetin and hydroxy citric acid on the status of lipid peroxidation and antioxidants in experimental non-alcoholic steatohepatitis. J Physiol Pharmacol 65: 67-74, 2014.

7. Doehner W and Landmesser U: Xanthine oxidase and uric acid in cardiovascular disease: Clinical impact and therapeutic options. Semin Nephrol 31: 433-440, 2011.

8. Sumida Y, Niki E, Naito Y and Yoshikawa T: Involvement of free radicals and oxidative stress in NAFLD/NASH. Free Radic Res 47: 869-880, 2013.

9. Mackness M and Mackness B: Targeting paraoxonase-1 in atherosclerosis. Expert Opin Ther Targets 17: 829-837, 2013.

10. Wang M, Lang X, Cui S, Zou L, Cao J, Wang S and Wu X: Quantitative assessment of the influence of paraoxonase 1 activity and coronary heart disease risk. DNA Cell Biol 31: 975-982, 2012.

11. Manning PJ, Jong SA, Ryalls AR and Sutherland WH: Paraoxonase 1 activity in chylomicrons and VLDL: The effect of type 2 diabetes and meals rich in saturated fat and oleic acid. Lipids 47: 259-267, 2012.

12. Ferre N, Marsillach J, Camps J, Mackness B, Mackness M, Riu F, Coll B, Tous M and Joven J: Paraoxonase-1 is associated with oxidative stress, fibrosis and FAS expression in chronic liver diseases. J Hepatol 45: 51-59, 2006.

13. Marsillach J, Camps J, Ferré N, Beltran R, Rull A, Mackness B, Mackness M and Joven J: Paraoxonase-1 is related to inflammation, fibrosis and PPAR delta in experimental liver disease. BMC Gastroenterol 9: 3, 2009.

14. Samy W and Hassanian MA: Paraoxonase-1 activity, malondialdehyde and glutathione peroxidase in non-alcoholic fatty liver disease and the effect of atorvastatin. Arab J Gastroenterol 12: 80-85, 2011.

15. Karsen H, Binici I, Sunnetcioglu M, Baran AI, Ceylan MR, Selek S and Celik H: Association of paraoxonase activity and atherosclerosis in patients with chronic hepatitis B. Afr Health Sci 12: 114-118, 2012.

16. Mingyue Z, Bing W, Qilong D, Ruining Y and Hong Q: Study on the relationship between xanthine oxidase paraoxonase-1 and occurrence and development of nonalcoholic fatty liver disease. Chinese Hepatology 19: 323-328, 2014.

17. Tuyama AC and Chang CY: Non-alcoholic fatty liver disease. J Diabetes 4: 266-280, 2012.

18. Day CP and James OF: Steatohepatitis: A tale of two 'hits'? Gastroenterology 114: 842-845, 1998.

19. Hijona E, Hijona L, Arenas JI and Bujanda L: Inflammatory mediators of hepatic steatosis. Mediators Inflamm 2010: 837419, 2010. 
20. Stiuso P, Scognamiglio I, Murolo M, Ferranti P, De Simone C, Rizzo MR, Tuccillo C, Caraglia M, Loguercio C and Federico A Serum oxidative stress markers and lipidomic profile to detect NASH patients responsive to an antioxidant treatment: A pilot study. Oxid Med Cell Longev 2014: 169216, 2014.

21. Koruk M, Taysi S, Savas MC, Yilmaz O, Akcay F and Karakok M: Oxidative stress and enzymatic antioxidant status in patients with nonalcoholic steatohepatitis. Ann Clin Lab Sci 34: 57-62, 2004.

22. Parks DA and Granger DN: Xanthine oxidase: Biochemistry, distribution and physiology. Acta Physiol Scand Suppl 548: 87-99, 1986

23. Sarnesto A, Linder N and Raivio KO: Organ distribution and molecular forms of human xanthine dehydrogenase/xanthine oxidase protein. Lab Invest 74: 48-56, 1996.

24. Morita M, Ishida N, Uchiyama K, Yamaguchi K, Itoh Y, Shichiri M, Yoshida Y, Hagihara Y, Naito Y, Yoshikawa T and Niki E: Fatty liver induced by free radicals and lipid peroxidation. Free Radic Res 46: 758-765, 2012.

25. Rodrigo L, Hernández AF, López-Caballero JJ, Gil F and Pla A: Immunohistochemical evidence for the expression and induction of paraoxonase in rat liver, kidney, lung and brain tissue. Implications for its physiological role. Chem Biol Interact 137: 123-137, 2001.

26. Karlsson H, Kontush A and James RW: Functionality of HDL: Antioxidation and detoxifying effects. Handb Exp Pharmacol 224: 207-228, 2015.

27. Mackness MI, Arrol S and Durrington PN: Paraoxonase prevents accumulation of lipoperoxides in low-density lipoprotein. FEBS Lett 286: 152-154, 1991.

28. Marsillach J, Camps J, Beltran-Debón R, Rull A, Aragones G, Maestre-Martínez C, Sabench F, Hernández M, Castillo DD, Joven $\mathrm{J}$, et al: Immunohistochemical analysis of paraoxonases-1 and 3 in human atheromatous plaques. Eur J Clin Invest 41: 308-314, 2011

29. Gür M, Çaylı M, Uçar H, Elbasan Z, Şahin DY, Gözükara MY, Selek S, Koyunsever NY, Şeker T, Türkoğlu C, et al: Paraoxonase (PON1) activity in patients with subclinical thoracic aortic atherosclerosis. Int J Cardiovasc Imaging 30: 889-895, 2014.
30. Fu AL and Wu SP: Single intravenous injection of plasmid DNA encoding human paraoxonase-1 inhibits hyperlipidemia in rats. Biochem Biophys Res Commun 397: 257-262, 2010.

31. Rosenblat M, Vaya J, Shih D and Aviram M: Paraoxonase 1 (PON1) enhances HDL-mediated macrophage cholesterol efflux via the ABCA1 transporter in association with increased HDL binding to the cells: A possible role for lysophosphatidylcholine. Atherosclerosis 179: 69-77, 2005.

32. Gorąca A, Huk-Kolega H, Piechota A, Kleniewska P, Ciejka E and Skibska B: Lipoic acid-biological activity and therapeutic potential. Pharmacol Rep 63: 849-858, 2011.

33. Rahimifard M, Navaei-Nigjeh M, Baeeri M, Maqbool F and Abdollahi M: Multiple protective mechanisms of alpha-lipoic acid in oxidation, apoptosis and inflammation against hydrogen peroxide induced toxicity in human lymphocytes. Mol Cell Biochem 403: 179-186, 2015.

34. Park S, Karunakaran U, Jeoung NH, Jeon JH and Lee IK: Physiological effect and therapeutic application of alpha lipoic acid. Curr Med Chem 21: 3636-3645, 2014.

35. Carrier B, Wen S, Zigouras S, Browne RW, Li Z, Patel MS, Williamson DL and Rideout TC: Alpha-lipoic acid reduces LDL-particle number and PCSK9 concentrations in high-fat fed obese Zucker rats. PLoS One 9: e90863, 2014.

36. Huerta AE, Navas-Carretero S, Prieto-Hontoria PL, Martínez JA and Moreno-Aliaga MJ: Effects of $\alpha$-lipoic acid and eicosapentaenoic acid in overweight and obese women during weight loss Obesity (Silver Spring) 23: 313-321, 2015.

37. Leuschner UF, Lindenthal B, Herrmann G, Arnold JC, Rössle M, Cordes HJ, Zeuzem S, Hein J and Berg T; NASH Study Group: High-dose ursodeoxycholic acid therapy for nonalcoholic steatohepatitis: A double-blind, randomized, placebo-controlled trial. Hepatology 52: 472-479, 2010.

38. Perra A, Pibiri M, Sulas P, Simbula G, Ledda-Columbano GM and Columbano A: Alpha-lipoic acid promotes the growth of rat hepatic pre-neoplastic lesions in the choline-deficient model. Carcinogenesis 29: 161-168, 2008. 